\title{
A Spectrum of Colors: Investigating the Temporal Frequency Characteristics of the Human Visual System Using a System Identification Approach
}

\author{
Edmund C. Lalor, Richard B. Reilly Senior Member IEEE, Barak A. Pearlmutter and John J. Foxe
}

\begin{abstract}
Noise input signals are commonly used in both linear and nonlinear system identification of physiological systems. This method can be applied to electrophysiological analysis of the human visual system by controlling the modulation of the contrast of a checkerboard stimulus using a pre-computed noise waveform. In this study we describe how one can obtain an estimate of the linear impulse response of the visual system using noise waveforms. Furthermore, we examine the impulse responses obtained using noise signals with different frequency characteristics, in an attempt to investigate the temporal frequency characteristics of the human visual system. We show that noise signals with frequency content greater than $15 \mathrm{~Hz}$ are more effective at evoking these responses than those with little or no power at high frequencies.
\end{abstract}

\section{INTRODUCTION}

The visual evoked potential (VEP) [1], [2] has become a routinely used and extremely valuable tool in both research and clinical settings for the evaluation of visual sensory and perceptual processing. In clinical testing, the so-called transient VEP is typically evoked by the repeated presentation of a visual stimulus at a rate of less than or equal to two presentations per second and extracted from the electroencephalogram (EEG) using signal averaging. When evoked by such repetitive stimulation, it shows several distinct components (e.g. $\mathrm{C} 1, \mathrm{P} 1, \mathrm{~N} 1)$ with distinctive topographies over the occipital scalp. A major advantage of the VEP technique is its exquisite temporal resolution, limited only by the sampling rate of the measurement device.

White noise signals are commonly used in both linear and nonlinear system identification of physiological systems [3], [4]. By considering the brain in simplified form as a linear system, with isolated events as input and EEG as output, the average event related potentials (ERPs) can be said to approximate the system's time-domain impulse

This work was supported by the Irish Higher Education Authority (grant HEA-r9310 to RBR), Science Foundation Ireland (grant 00/PI.1/C067 to the Hamilton Institute at NUI Maynooth), and the US National Institute of Mental Health (grant NIMH-MH65350 to JJF)

E. C. Lalor and R. B. Reilly are with the School of Electrical, Electronic and Mechanical Engineering, University College Dublin, Belfield, Dublin 4, Ireland and the Cognitive Neurophysiology Laboratory, St Vincent's Hospital, Fairview, Dublin, Ireland ed.lalor@ee.ucd.ie richard.reilly@ucd.ie

B. A. Pearlmutter is with the Hamilton Institute, National University of Ireland Maynooth, Co. Kildare, Ireland barakecs . nuim. ie

J. J. Foxe is with the Program in Cognitive Neuroscience, Department of Psychology, The City College of the City University of New York, New York, USA and with the Cognitive Neurophysiology Laboratory, the Nathan Kline Instite for Psychiatric Research, Orangeburg, New York Foxe@NKI.RFMH. ORG response functions, which is the linear part of the eventrelated dynamics. However, in reality, events are not isolated. Rather, inputs occur in a rapid and continuous stream and their associated electrophysiological responses often overlap in time. Consequently, an ERP calculated as the average of many isolated inputs may obscure the brain's response dynamics.

The impulse response of the visual system to a stimulus, whose luminance or contrast is modulated by a waveform with uniform power over the range $0-30 \mathrm{~Hz}$ can be obtained using linear least squares estimation [5]. This response is known as the visual evoked spread spectrum response or VESPA. While a stimulus waveform with these statistics is effective at activating the early visual system, as evidenced by the signal-to-noise ratios (SNR) of the VESPA over area V1, improvements might be attained by using an input waveform with more complex statistics.

In particular, the frequency characteristics of the VESPA obtained by using a uniform power stimulus gives an indication of the characteristics of the visual system itself. This information, combined with prior information about noisy subsystems within the visual cortex e.g. the alpha band, suggests that VESPAs with improved SNR might be obtainable.

A rapidly changing stimulus might be effective at isolating the dorsal stream of the visual system. This could facilitate rapid assessment of this subsystem, a procedure which is slow and laborious using current methods. This can be tested by shaping the spectrum of the input waveform so that most of the power is in the higher frequencies of the 0 $30 \mathrm{~Hz}$ band. Using this waveform, the ability of such spread spectrum stimuli to isolate the magnocellular pathways can be assessed, at least insofar as an EEG study will allow.

Finally, stimuli with considerable energy both inside and outside the $8-12 \mathrm{~Hz}$ alpha range are investigated. While the large amount of spontaneous activity within this range of frequencies suggests that using such a stimulus could result in a lower SNR, it is hoped that the stimulus may "drive" the alpha band activity, resulting in better performance.

\section{METHODS}

\section{A. Subjects}

Three subjects (one female) aged 28-41 participated in the study. All had normal or corrected-to-normal vision. 


\section{B. Hardware}

Subjects were seated $60 \mathrm{~cm}$ from a 19 in computer monitor driven by an NVIDIA GeForce FX5200 video card, at a refresh rate of $60 \mathrm{~Hz}$. EEG data were recorded from 64 electrode positions referenced to fronto-central location $\mathrm{Fz}$, filtered over the range $0-134 \mathrm{~Hz}$ and digitized at a rate of $512 \mathrm{~Hz}$ using a BioSemi Active Two system. Synchronization between the video display and the EEG signals was ensured by including the signal on the parallel port of the presentation computer, controlled by the presentation software, among the signals acquired by the analog-to-digital converter bank.

The EEG was digitally filtered with a high-pass filter with a passband above $2 \mathrm{~Hz}$ and a $-60 \mathrm{~dB}$ response at $1 \mathrm{~Hz}$ and a low-pass filter with a $0-35 \mathrm{~Hz}$ passband and a -50 $\mathrm{dB}$ response at $45 \mathrm{~Hz}$. The response properties of the video monitor used for stimulus presentation were measured using a Nuclear Associates photometer, model 07-621, with an ambient light shield.

\section{Images}

The basic image used in this study was a standard checkerboard pattern. This consisted of equal numbers of black and white checks. Each check subtended a visual angle of $0.65^{\circ}$ both horizontally and vertically, while the checkerboard as a whole subtended visual angles of $5.25^{\circ}$ vertically and horizontally. The luminances of the black and white checks were measured as $0.1 \mathrm{~cd} / \mathrm{m}^{2}$ and $164 \mathrm{~cd} / \mathrm{m}^{2}$ respectively, giving a Michelson contrast of $99.9 \%$.

Using the measure monitor response profile, 68 checkerboards were generated where the mean of the luminance of the lighter checks and the darker checks were approximately equal for each checkerboard. For example, the checkerboard consisting of dark checks of gray-scale level 0 and light checks of gray-scale level 255 has a mean luminance of approximately $82 \mathrm{~cd} / \mathrm{m}^{2}$. Similarly, the checkerboard consisting of dark checks of gray-scale level 129 and light checks of gray-scale level 230 has a mean luminance of approximately $82 \mathrm{~cd} / \mathrm{m}^{2}$. Finally, the uniform image consisting of pixels at gray-scale level 188 also has a mean luminance of $82 \mathrm{~cd} / \mathrm{m}^{2}$. Examples of the images used can be seen in Fig. 1.

\section{Spread Spectrum Waveforms}

For all of the spread spectrum stimuli, the image being displayed is controlled directly by an underlying spread spectrum modulation waveform. Using the Nyquist sampling theorem and given that EEG power above $30 \mathrm{~Hz}$ is very low, the monitor refresh rate was set to $60 \mathrm{~Hz}$. This underlying
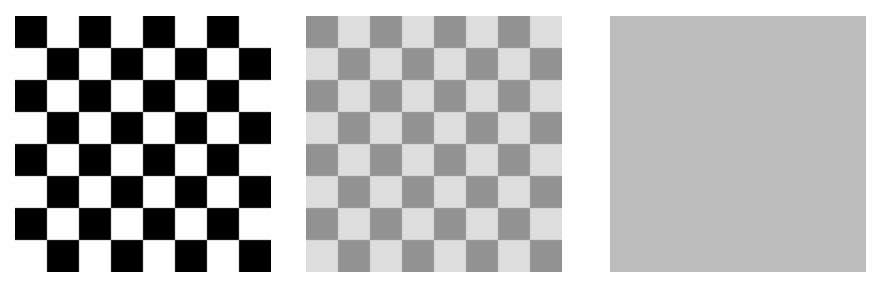

Fig. 1. Constant mean luminance checkerboards 0, 34 and 67. spread spectrum waveform was mapped to the checkerboard images according to a linear relation, with the zero-point of the waveform corresponding to checkerboard 34, and scaled to allow \pm three standard deviations within the range of the images. On every refresh of the computer monitor, the checkerboard image corresponding to the current sample of the input waveform was displayed. The EEG data was tagged with the value of the luminance of the light checks minus the luminance of the dark checks. Fig. 1 shows three of these mean luminance checkerboards.

Waveforms with any desired statistical properties can be pre-computed and stored. This is accomplished by first choosing a target power spectrum, then shaping Gaussian noise appropriately. Six different filters were used in this study to investigate the pass through characteristics of the visual system.

1) White: The first set up that was investigated used a spread spectrum waveform with uniform power in the interval $0-30 \mathrm{~Hz}$ as shown in Fig. 2(a).

2) Damped Alpha: Taking into account the fact that there is a large amount of spontaneous EEG activity in the alpha band (i.e. 8-12 Hz), we used a shaping filter where we scaled down the coefficients corresponding to frequencies in this range. The shaping filter used can be seen in Fig. 2(b).

3) Alpha Band: The shaping filter shown in Fig. 2(c) was used to investigate the quality of the impulse response obtained by using a stimulus with most of its power in the $8-12 \mathrm{~Hz}$ range. As mentioned, there is a large amount of spontaneous EEG activity in this range and it was considered worth investigating whether this EEG could be "driven" by the stimulus, thereby taking advantage of the high alpha power.

4) High Pass: In an effort to investigate the response to high frequency stimuli the shaping filter shown in Fig. 2(d) was used.

5) VESPA: The power spectrum of the average VESPA obtained using the mean luminance checkerboard stimulus modulated by the waveform with uniform power over the 0-30 Hz range was calculated and is shown in Fig. 2(e). It was felt that this would give a good indication of which frequencies were processed well by the visual system and which were not and that by shaping the spectrum of the input waveform accordingly we might improve the SNR. Therefore we generated Gaussian colored noise with the same profile as the p.s.d. of the VESPA as plotted in Fig. 2(e).

6) VESPA with damped alpha: Finally, we investigated the efficacy of using a waveform which was filtered based on the spectrum of the original VESPA with a scaling down of the power in the alpha band. This filter is shown in Fig. 2(f).

\section{E. Experimental Procedure}

Subjects were instructed to maintain visual fixation on the centre of the screen for the duration of each testing session. While abstaining from eye-blinks was not possible given the session lengths, subjects were instructed to keep the number of eye-blinks to a minimum during each session. Subjects 

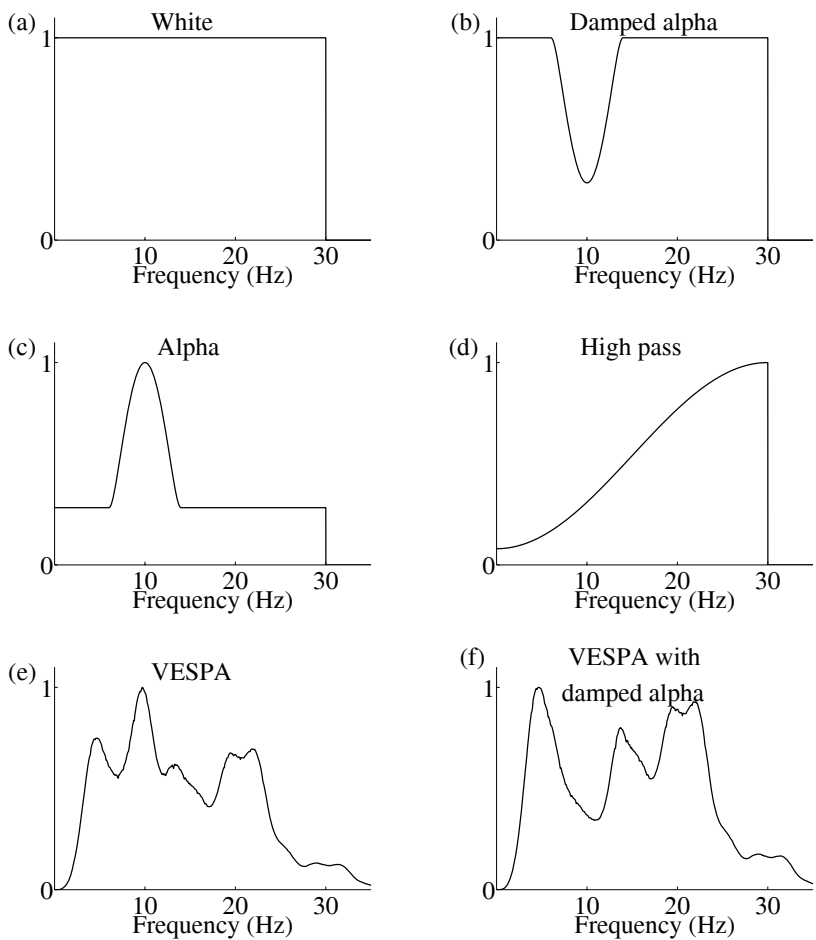

Fig. 2. The six shaping filters investigated in this study.

were also instructed to keep all other types of motor activity to a minimum during testing.

Each subject undertook two sessions of 120 seconds each for the spread spectrum stimulus using mean luminance checkerboards for each type of shaping filter. This gave a total of 12 two-minute trials.

The order of presentation of stimuli modulated by waveforms with different statistics was counterbalanced between subjects, and no set up was ever undertaken twice in succession.

\section{F. Signal Processing}

We obtain the VESPA by performing a linear least squares fit of the response model

$$
y(t)=w(\tau) * x(t)+\text { noise }
$$

where $y(t)$ is the measured EEG response, $x(t)$ is the intensity waveform of the stimulus, the symbol $*$ indicates convolution, $w(\tau)$ is the impulse-response function to the brightness of the stimulus, and noise is assumed to be Gaussian [5]. The values of $x_{t}$ are taken to be the difference in luminance between the light and dark checks of each checkerboard.

\section{G. Quantification of Performance}

SNRs were calculated for the VESPAs corresponding to each different shaping filter. These SNRs were calculated by defining the noise as the mean of the squared values in the $100 \mathrm{~ms}$ interval immediately preceding the stimulus, and the
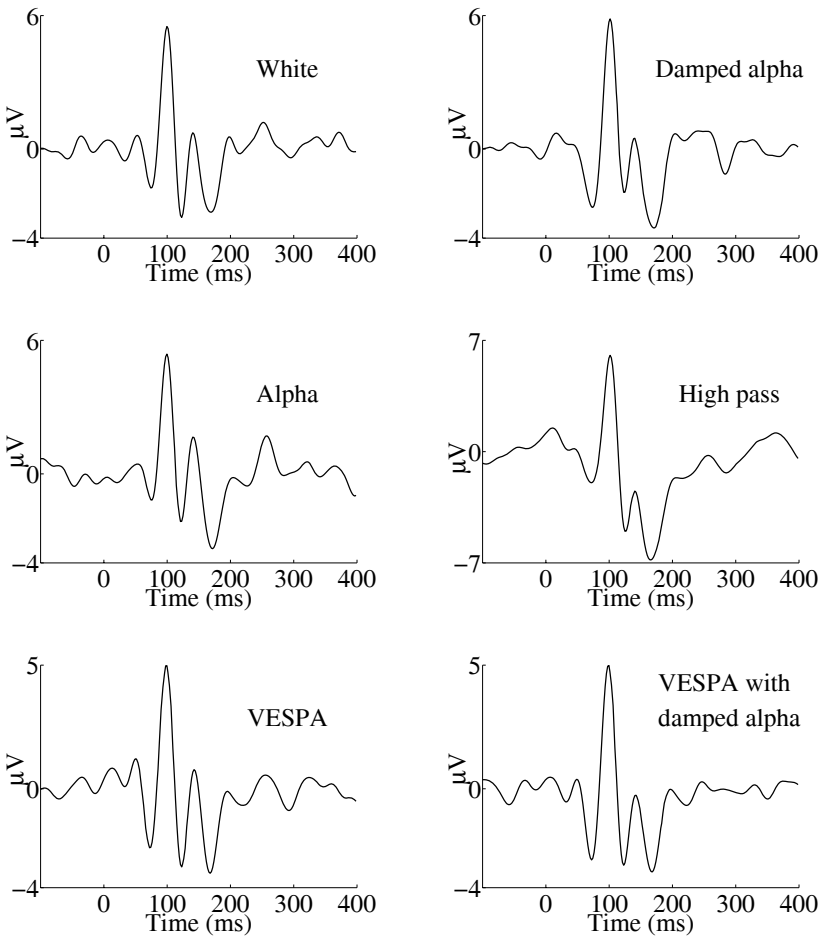

Fig. 3. The VESPA impulse responses measured using each of the six different stimulation waveforms.

signal was defined as the mean of the squared values in the interval 35-175 ms post-stimulus.

The SNR of each VESPA was calculated at occipitocentral electrode location $\mathrm{Oz}$ at 5 second intervals for each subject and each session and then averaged across subjects and sessions.

\section{RESULTS}

Fig. 3 shows the VESPA impulse responses to the six different stimulation waveforms.

Fig. 4 shows a plot of the SNR of the impulse responses, calculated at 5 second intervals, for each of the shaping filters and averaged across subjects and sessions. As can be seen from the plot, the high pass filter gives the best SNR after 120 seconds having an SNR of over $17 \mathrm{~dB}$ compared to the $16.4 \mathrm{~dB}$ for the white noise waveform. Interestingly, the case where the alpha is heavily damped and the case where everything but the alpha is heavily damped have very similar SNR after 120 seconds at $15.4 \mathrm{~dB}$ and $14.6 \mathrm{~dB}$ respectively. Finally, the cases where the spectrum was shaped using the spectrum of the VESPA response to the white noise stimulus had the poorest SNRs. The SNR value after 120 seconds for the stimulus based on the VESPA was $9.2 \mathrm{~dB}$. A slightly higher SNR of $11 \mathrm{~dB}$ was obtained in the case where the alpha power was damped.

\section{DISCUSSION}

While others have analyzed the frequency characteristics of the visual system in electrophysiological studies using single frequency stimuli [6], [7], we have shown that it 


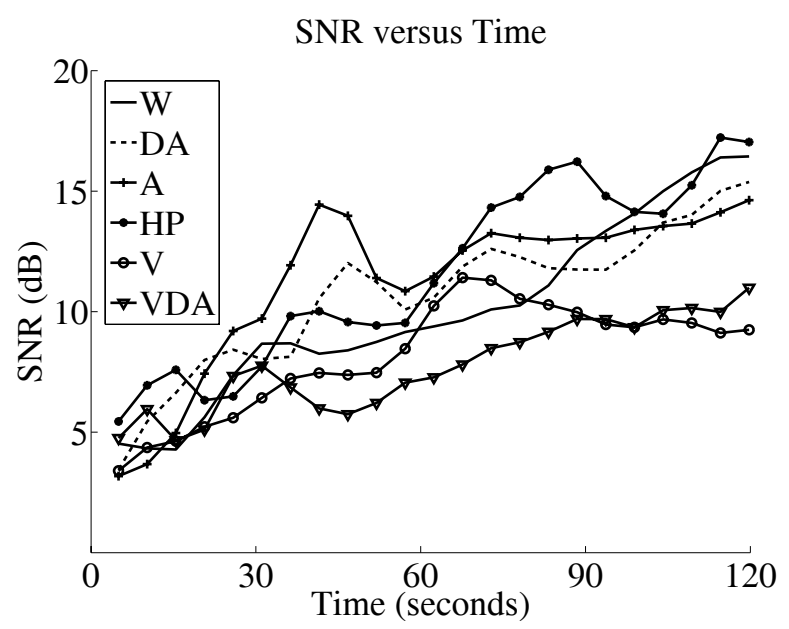

Fig. 4. SNR at 5 second intervals for each of the shaping filters averaged across subjects and sessions. 'W', 'DA', 'A', 'HP', 'V', 'VDA' represent the white, damped alpha, alpha, high pass, VESPA, and VESPA with damped alpha filters, respectively.

is possible to rapidly obtain impulse responses to stimuli modulated with high SNRs using spread spectrum waveforms with different statistics.

The plots in Fig. 3 and the SNR curves in Fig. 4 illustrate that shaping the spectrum based on the VESPA obtained by stimulating with a white noise stimulus does not improve the SNR and, in fact, results in the two poorest SNR curves. Perhaps a more complex mapping from the VESPA p.s.d. to the input waveform statistics would result in an improvement. For example, consider allocating power in the input waveform as a function of the ratio of noise-to-gain at each frequency bin for the original VESPA. This would put more power into driving those frequency bands that performed poorly and less into the frequency bands that don't need to be driven as hard. The plots also show that the spontaneous alpha band activity associated with EEG has had neither a positive nor a particularly negative effect on the estimation of the impulse response. Finally, the highpass filtered stimulus and the white noise stimulus give the best SNR curves. This suggests that stimuli with power in the higher frequencies activate the visual system most effectively using the spread spectrum stimulation method.

The VESPA tends to be very focused over the midline [5]. This suggests that the spread spectrum stimulation particularly targets the dorsal visual stream, which is thought to respond to rapidly changing stimuli and motion. The effectiveness of the stimuli with power in the high frequencies, i.e. the most rapidly changing stimuli, lends support to this hypotheses. Conveniently, the most rapidly changing stimuli are in fact the least visually obtrusive (consider the difference between a light flickering at $15 \mathrm{~Hz}$ and one flickering at 60 $\mathrm{Hz}$ !)

Differences in the scalp topographies of the VESPAs to the different simulation waveforms were investigated. Scalp maps were plotted for the VESPA responses at 75, 100,
110, 130 and $155 \mathrm{~ms}$ (not shown). These indicated that the topographies were very similar for the different waveforms. More subjects would be needed in order to test whether any small differences in the topographies are significant. The poor spatial resolution of EEG may simply not be sufficient to pick up subtle differences in the activation patterns of cell subpopulations.

Recent research has identified changes in certain components of the VEP in patients with schizophrenia that may occur as a result of deficits specific to the dorsal visual stream [8]. It is also suggested that certain ventral stream processes are contingent on inputs from the dorsal stream, and as a result failure in these 'higher-level' ventral stream processes may be caused by these underlying dorsal stream deficits. Since the spread spectrum stimulation method with high frequency stimuli seems to effectively isolate the dorsal visual stream, and that they allow for the estimation of VESPAs with distinct components, the VESPA may have a significant and immediate impact on research into schizophrenia.

\section{CONCLUSIONS}

Impulse responses, known as VESPAs, can be rapidly estimated using spread spectrum stimuli with different frequency characteristics. It appears that stimuli with power at higher frequencies more effectively activates the neural pathways associated with this method.

A schizophrenia study using the spread spectrum method with white noise stimuli is underway.

An fMRI/DTI study showing which neural pathways are activated using spread spectrum stimuli with different frequency characteristics might provide more explanation for the results shown in this study. A more comprehensive EEG study using more subjects and other types of shaping filters would also be useful.

\section{REFERENCES}

[1] W.A. Cobb and G.D. Dawson, The latency and form in man of the occipital potentials evoked by bright flashes, Journal of Physiology, vol. 152,1960 , pp 108-121.

[2] H.G. Vaughan Jr. and R.C. Hull, Functional relation between stimulus intensity and photically evoked cerebralresponses in man, Nature, vol. 206(985), 1965, pp 720-722.

[3] P.Z. Marmarelis and V.Z. Marmarelis, Analysis of Physiological Systems: The White Noise Approach, Plenum Press, 1978.

[4] Richard Coppola, A System Transfer Function for Visual Evoked Potentials, Human Evoked Potentials: Applications and Problems, Plenum Press, 1979, pp 69-82.

[5] E.C. Lalor, B.A. Pearlmutter, R.B. Reilly, G. McDarby and J.J. Foxe, The VESPA: A Method for the Rapid Estimation of a Visual Evoked Potential, NeuroImage, In press.

[6] C.S. Herrmann, Human EEG responses to $1100 \mathrm{~Hz}$ flicker: resonance phenomena in visual cortex and their potential correlation to cognitive phenomena, Experimental Brain Research, vol. 137, 2001, pp 346353.

[7] I.P. Fawcett, G.R. Barnes, A. Hillebrand, and K.D. Singh, The temporal frequency tuning of human visual cortex investigated using synthetic aperture magnetometry, NeuroImage, vol. 21, 2004, pp 15421553.

[8] J.J. Foxe, M.M. Murray and D.C. Javitt, Filling-in in Schizophrenia: a High-density Electrical Mapping and Source-analysis Investigation of Illusory Contour Processing, Cerebral Cortex, vol. 15, 2005, pp 1914-1927 